\title{
Adaptação transcultural, confiabilidade e validade da escala de resiliência
}

\author{
Cross-cultural adaptation, reliability \\ and validity of the resilience scale
}

\author{
1 Centro Latino-Americano \\ de Estudos de Violência \\ e Saúde Jorge Careli, \\ Fundação Oswaldo Cruz, \\ Rio de Janeiro, Brasil. \\ 2 Escola Nacional de Saúde \\ Pública, Fundação Oswaldo \\ Cruz, Rio de Janeiro, Brasil. \\ Correspondência \\ R. P. Pesce \\ Centro Latino-Americano \\ de Estudos de Violência \\ e Saúde Jorge Careli, \\ Fundação Oswaldo Cruz. \\ Av. Brasil 4036, \\ Rio de Janeiro, $R J$ \\ 21040-361, Brasil. \\ rpesce@claves.fiocruz.br
}

\begin{abstract}
This study describes the cross-cultural adaptation to Portuguese and the psychometric evaluation of the resilience scale developed by Wagnild \& Young. The scale was adapted for a sample of students from public schools in São Gonçalo, Rio de Janeiro, Brazil. Data from the pilot study (203 students interviewed at two points in time) and from the entire study (977) are presented. The cross-cultural adaptation showed good results in the semantic equivalence for: general meaning (above 90.0\%) and referential meaning (above 85.0\%). Chronbach alpha was 0.85 in the pilot study and 0.80 in the total sample. Kappa between the two points in time was regular and moderate, and the intraclass correlation coefficient was $0.746(p=0.000)$. Factorial analysis indicated three non-homogeneous factors. Construct validity demonstrated direct and significant correlation with self-esteem, family supervision, life satisfaction, and social support. There was an inverse correlation with the scale that evaluates psychological violence.
\end{abstract}

Adaptation; Reproducibility of Results; Questionnaires

\author{
Renata P. Pesce 1,2 \\ Simone G. Assis 2 \\ Joviana Q. Avanci 2 \\ Nilton C. Santos 2 \\ Juaci V. Malaquias ${ }^{2}$ \\ Raquel Carvalhaes ${ }^{2}$
}

\section{Introdução}

O contemporâneo movimento pela promoção da saúde tem revelado a resiliência como um conceito importante nessa área de conhecimento, especialmente no campo da criança e do adolescente. Por ser um tema recentemente incorporado ao campo da saúde, encontra-se em fase de construção, discussão e debate, principalmente por não existir ainda um consenso em relação à definição do termo. Por resiliência entende-se o conjunto de processos sociais e intrapsíquicos que possibilitam o desenvolvimento saudável do indivíduo, mesmo este vivenciando experiências desfavoráveis. A complexidade do constructo envolve a interação entre eventos de vida adversos e fatores de proteção internos e externos ao indivíduo 1,2 .

Estudos com crianças resilientes apontam três tipos de variáveis que operariam como fatores de proteção à adversidade: (a) características de personalidade, como a auto-estima, flexibilidade, habilidade para resolução de conflitos; (b) coesão e bom relacionamento na família; (c) disponibilidade de suporte externo que encoraje e reforce as estratégias de coping da criança, especialmente provenientes do grupo de pares, escola e comunidade 1,3 .

O crescente interesse pelo conceito de resiliência como reflexo da necessidade do investimento em prevenção de problemas e promoção da saúde mental torna importante a exis- 
tência de instrumentos para avaliação desse constructo. Poucas escalas abordando esse tema são relatadas na literatura 4,5,6,7. No Brasil, ainda não há nenhuma criada ou adaptada.

A escala de resiliência desenvolvida por Wagnild \& Young 8 é um dos poucos instrumentos usados para medir níveis de adaptação psicossocial positiva em face de eventos de vida importantes. Possui 25 itens descritos de forma positiva com resposta tipo likert variando de 1 (discordo totalmente) a 7 (concordo totalmente). Os escores da escala oscilam de 25 a 175 pontos, com valores altos indicando elevada resiliência. O instrumento foi desenvolvido por meio de um estudo qualitativo com $24 \mathrm{mu}$ lheres adultas previamente selecionadas por adaptarem-se com sucesso à adversidade da vida. Cada uma delas foi solicitada a descrever como se organizavam diante de vivências negativas. De suas narrativas, cinco componentes foram identificados como fatores para resiliência: serenidade, perseverança, autoconfiança, sentido de vida e auto-suficiência. As colocações verbais das participantes foram validadas e esclarecidas mediante revisão bibliográfica do tema, concluindo-se que a escala de resiliência possui $a$ priori validade de conteúdo, pois seus itens refletem a aceitação geral das definições de resiliência. Estudos iniciais obtiveram bons indicativos de confiabilidade e validade desse instrumento, conforme citado em Wagnild \& Young ${ }^{8}$.

Todavia, para que um instrumento possa ser utilizado em outros contextos, recomenda-se um rigoroso processo de adaptação e análise dos índices psicométricos, já que somente a adaptação semântica não cobre as diferenças culturais entre os dois universos explorados, além de prejudicar a análise dos resultados de validade e confiabilidade da escala 9,10,11,12,13,14,15.

O presente artigo tem o objetivo de explicitar os procedimentos efetuados na adaptação transcultural e avaliação psicométrica da escala de resiliência desenvolvida por Wagnild \& Young 8 , valendo-se de uma amostra de escolares de $7 \underline{a}$ e $8 \underline{a}$ séries do ensino fundamental e 1o e 2 o anos do ensino médio da rede pública de ensino do Município de São Gonçalo, no Rio de Janeiro, Brasil. Por meio do processo de comparação entre o instrumento original e sua versão para o português, pretende-se contribuir para a avaliação da equivalência transcultural entre os instrumentos.

\section{Materiais e métodos}

\section{População e amostra}

O plano amostral deste trabalho baseou-se na população de alunos matriculados em escolas públicas do Município de São Gonçalo, divididos em dois estratos, segundo a série cursada em 2002 (7âa/8 a do ensino fundamental e 1aa $2 \underline{a}$ do ensino médio), de acordo com informações fornecidas pela Secretaria de Educação do Município.

Em virtude da dificuldade para conseguir um cadastro de alunos individual e também pela falta de viabilidade prática na execução de uma seleção aleatória de alunos, foi realizada uma amostragem conglomerada para a escolha das escolas. O procedimento de seleção passou por duas etapas: (a) escolha das escolas (representantes do conjunto de turmas e alunos dos estratos selecionados); (b) seleção das turmas por amostragem aleatória simples dentro das escolas. Em cada turma selecionada, todos os alunos que estavam presentes no dia da aplicação do questionário responderam a ele.

As unidades primárias de amostragem foram as escolas, o que possibilitou a participação de um mesmo estabelecimento de ensino nos universos dos dois grupos de série, desde que este tivesse turmas e alunos em mais de um dos grupos de séries consideradas. Selecionaram-se escolas usando amostragem com probabilidades proporcionais ao número de turmas dentro de cada estrato de tamanho. Para produzir uma maior sobreposição entre as unidades escolares nas amostras dos dois grupos de séries, decidiu-se utilizar o método denominado Amostragem Seqüencial de Poisson 16. Para escolas com duas ou mais turmas, efetuouse amostragem de turmas, a fim de garantir uma distribuição proporcional entre as escolas maiores e menores e entre as turmas dos turnos matinal e vespertino.

Dentre as 88 escolas de $7 \underline{a}$ e $8 \underline{a}$ séries (510 turmas; 17.133 alunos), foram sorteados, para o estudo, 500 alunos, dos quais se entrevistou um total de 485 . No que se refere às 43 escolas de 1 o e 2 o anos (263 turmas; 10.841 alunos), obteve-se um total de 512 entrevistados dentre os 500 inicialmente selecionados para comporem a amostra, superando a estimativa inicial. Foram entrevistados 997 alunos entre 12 e 19 anos (média $=15,4 ; \mathrm{DP}=1,48$ ), com supremacia de meninas $(57,7 \%)$, nas 11 escolas de $7 \underline{a}$ e $8 \underline{a}$ séries e nas 10 escolas de 10 e $2 \underline{o}$ anos visitadas. 
No total, 17 diferentes escolas (4 escolas com os dois segmentos) e 37 turmas participaram do estudo.

Uma amostra não probabilística reduzida dessa população participou da etapa de teste-reteste do instrumento. Foram entrevistados 203 alunos em três escolas do município (idade média $=14,6 ; \mathrm{DP}=1,93 ; 52,2 \%$ do sexo feminino).

\section{Adaptação transcultural}

Das diferentes formas de adaptação, optou-se pelo método proposto por Herdman et al. $12 \mathrm{e}$ divulgado por Hasselmann \& Reichenheim $13 \mathrm{e}$ Moraes et al. 15, que consta de uma lista de passos a serem seguidos até a aceitação de equivalência entre instrumentos.

Equivalência conceitual: refere-se à equivalência do conceito na cultura original em comparação à cultura-alvo. No presente estudo, o primeiro passo dado foi a exaustiva revisão bibliográfica sobre o tema na população-alvo e na original. A avaliação de especialistas na área contribuiu para a conclusão de que o domínio do instrumento original é pertinente ao conhecimento sobre resiliência, ainda muito restrito no meio científico e, em especial, no Brasil.

Equivalência de itens: indica se os itens que compõem a escala de resiliência estimam os mesmos domínios e se são relevantes nas duas culturas. Envolve as mesmas etapas da equivalência conceitual - revisão da literatura, consulta com especialistas e com a população-alvo. Na versão para o português, verificou-se se existiam itens que devessem ser substituídos por não terem a mesma conotação do termo original. Foi também avaliada a consistência interna da escala estimada por intermédio do alpha de Cronbach tanto no pré-teste, quanto no estudo global. Essa técnica de mensuração fornece uma idéia mais real da relevância dos itens, indicando o quanto estes estão medindo um determinado conceito 17.

Equivalência semântica: consiste na tradução do instrumento original não só conservando o significado das palavras entre dois idiomas diferentes, como também buscando atingir o mesmo efeito em culturas distintas. Consistiu em cinco etapas: (1) foram feitas duas traduções de forma independente, da escala em inglês para o português, por profissionais com conhecimento da língua inglesa (graduação em Letras e psicóloga com formação em inglês); (2) foram efetuadas duas retraduções, também de forma independente, por outros dois profissionais familiarizados com a língua e a cultura inglesa; (3) um quinto profissional com profundo conhecimento do idioma e da cultura ingle- sa avaliou a concordância entre os itens originais e as duas retraduções. Essa avaliação foi feita de forma "cega", para que não se percebesse qual o item original e qual sofreu tradução/retradução. Duas categorias de equivalência semântica entre os itens foram avaliadas por esse profissional: significado referencial, que corresponde à concordância em termos de tradução literal entre um item original e o mesmo retraduzido, avaliado pela Visual Analogue Scale, em que a equivalência entre pares é julgada de 0 a $100 \%$ 17; significado geral, correspondente à concordância mais ampla, em termos de articulação de idéias e impacto entre um item original e sua retradução, avaliada conforme quatro níveis de equivalência: inalterado, pouco alterado, muito alterado ou completamente alterado. O profissional responsável por essa etapa fez comentários e forneceu sugestões para que os itens retraduzidos ficassem o mais próximo possível de seus originais; (4) os autores do presente artigo avaliaram as apreciações feitas pelos especialistas, discutindo os itens e decidindo por aqueles que comporiam a nova versão da escala para o português; (5) aplicação da versão final da escala aos adolescentes escolares, na fase de pré-teste do instrumento, buscando-se observar se as questões foram bem compreendidas pela população-alvo do estudo.

Equivalência operacional: visa a manter características operacionais do universo original, propiciando maior confiabilidade e validade do instrumento, por intermédio de medidas empregadas antes e durante a aplicação da escala, tais como: mesmo número de questões (25 questões); mesmas sete opções de respostas ordinais utilizadas no instrumento original; questionário auto-administrado acompanhado por duas psicólogas treinadas, com tempo médio de sessenta minutos por turma; treinamento dos aplicadores, tendo como base a elaboração de um instrumento para orientar o preenchimento do questionário pelos alunos.

Equivalência de mensuração: refere-se às propriedades psicométricas utilizadas para testar a equivalência de um instrumento em duas línguas diferentes, avaliada por meio de medidas de confiabilidade e validade.

Mediu-se a confiabilidade intra-observador mediante um estudo no qual os adolescentes responderam ao questionário em dois momentos distintos, chamados de momento 0 (teste) e momento 1 (reteste), em intervalo de dez dias entre as aplicações. Para a comparação dos dois momentos, utilizou-se o coeficiente de correlação intraclasse para variáveis contínuas e a estatística kappa ponderado para a análise 
dos dados com variáveis de escala ordinal. Essa última estatística leva em consideração a discordância parcial, ao atribuir pesos diferentes aos graus distintos de discordância. As respostas discordantes foram ponderadas pelos quadrados dos desvios da concordância simples, permitindo uma interpretação equivalente ao coeficiente de correlação intraclasse. Os critérios adotados para a interpretação do grau de concordância foram (a) concordância quase perfeita: 0,80 a 1,00; (b) concordância substancial: 0,60 a 0,80; (c) concordância moderada: 0,40 a 0,60; (d) concordância regular: 0,20 a 0,40; (e) concordância discreta: 0,00 a 0,20 ; (f) concordância pobre: $-1,00$ a 0,00 18 .

Outro indicativo de confiabilidade calculado foi o Alpha de Chronbach, para verificar a consistência interna do instrumento na fase do pré-teste e na amostra total.

A validade de conteúdo refere-se ao grau em que o conteúdo ou os domínios de um instrumento de mensuração são relevantes e representativos do constructo-alvo para um objetivo particular de avaliação 17. Essa forma de validade foi medida por intermédio de um julgamento teórico realizado por três profissionais especialistas no tema. No que se refere à validade de constructo, foi feita uma demonstração de que o instrumento mede aquilo que se propõe a medir, fazendo a correlação com outros conceitos que, teoricamente, são relevantes em relação ao tema que o instrumento se propõe a medir. Wagnild \& Young 8 encontraram elevada correlação da resiliência com constructos como satisfação na vida, saúde física e baixo nível de depressão.

No presente estudo, a escala de resiliência foi correlacionada com alguns constructos teoricamente relevantes na literatura sobre o tema: supervisão familiar, auto-estima, satisfação de vida, apoio social e violência psicológica. Os conceitos citados foram mensurados, respectivamente, da seguinte forma: três itens independentes abordando supervisão dos pais, escala de auto-estima de Rosenberg 19, escala de satisfação de vida 20 , escala de apoio social 21 e escala de violência psicológica 22 . Essas escalas mostraram bons índices psicométricos na mesma população estudada neste artigo 23.

A análise fatorial dos itens de uma escala consiste em uma técnica estatística multivariada que tenta reduzir a dimensionalidade mediante diferentes estratégias de rotação. Nesta pesquisa, foram utilizadas as rotações oblíqua e ortogonal, que quase sempre produzem resultados similares. A primeira correlaciona os fatores entre si e é utilizada em estudos exploratórios, quando se pretende obter resultados que melhor se ajustem à amostra do estudo. A segunda, utilizada na maioria dos estudos, não correlaciona os fatores uns com os outros, assim os resultados oferecidos são mais generalizáveis e, portanto, mais replicáveis 24 .

A primeira análise fatorial da escala de resiliência foi feita por seus autores, Wagnild \& Young 8 , com dados de uma amostra de 810 adultos idosos. Nessa análise, efetuou-se a rotação oblíqua, encontrando cinco fatores de solução; no entanto, parando a produção dos fatores quando o último fator explicou não menos que 5,0\% da variância, dois fatores de solução foram encontrados e provaram ser mais interpretáveis. Os 17 itens do fator I, denominado "competência pessoal", sugerem autoconfiança, independência, determinação, invencibilidade, controle, desenvoltura e perseverança. Os oito itens do fator II, denominado "aceitação de si mesmo e da vida”, representam adaptabilidade, equilíbrio, flexibilidade e perspectiva de vida equilibrada. Todos os loadings dos itens foram 0,40 ou maior. O total de 44,0\% da variância foi explicado pela solução de dois fatores.

Na presente pesquisa, foi aplicada a rotação oblíqua aos itens respondidos pela amostra total do estudo com objetivo de comparar os resultados obtidos com aqueles encontrados por Wagnild \& Young ${ }^{8}$. De forma complementar, utilizou-se a rotação ortogonal para proporcionar uma solução de fatores que melhor se ajustasse a dados passados e futuros. Em ambos os casos, optou-se por parar a produção dos fatores ao se atingirem $5,0 \%$ da variância.

Equivalência funcional: síntese das demais formas de equivalência, a qual permite afirmar se uma escala pode ser aplicada na nova população-alvo.

\section{Processamento dos dados}

O banco de entrada de dados foi criado no programa Epi Info 6.0, cumprindo quatro rigorosas etapas durante o processamento: codificação, digitação, correção e análise. Apenas $0,2 \%$ das questões apresentou erros de digitação. Inconsistências entre questões, decorrentes de má interpretação destas pelo entrevistado, levaram à anulação de $0,6 \%$ delas. Na fase de análise, os bancos foram convertidos para o programa SPSS versão 8.0.

\section{Resultados}

No que se refere à avaliação da equivalência conceitual e de itens, a revisão bibliográfica sobre o tema e a discussão e debate entre profis- 
sionais especializados na área apontaram para a pertinência do instrumento original na nossa cultura. As dimensões sugeridas pelos autores da escala, indicando "competência pessoal" e "aceitação de si mesmo e da vida", foram consideradas pertencentes às definições teóricas e conceituais da resiliência.

Optou-se pela conservação dos 25 itens originais, buscando mantê-los em seus sentidos originais. Alguns itens da escala proposta por Wagnild \& Young 8 foram considerados de difícil tradução, por possuírem termos e expressões não comuns. Dois itens em especial foram mais discutidos entre os profissionais responsáveis pelo processo de adaptação da escala, exigindo um esforço maior de compreensão. $\mathrm{Na}$ versão-síntese proposta, o item 7 , originalmente elaborado como "I usually take things in stride", foi representado por "eu costumo aceitar as coisas sem muita preocupação”; o item 12, formulado originalmente como "I take things one day at a time", foi transformado em "eu faço as coisas um dia de cada vez". Uma outra questão dividiu a equipe por causa da interpretação positiva dada pelos autores: "I seldom wonder what the point of all is" (item 11), traduzida como "eu raramente penso sobre o objetivo das coisas". Esses obstáculos refletem tanto as dificuldades de se lidar com um conceito pouco explorado, quanto as diferenças culturais.

Quanto à avaliação da equivalência semântica, observou-se que o significado referencial apresentou bons resultados, todos os itens obtendo, em uma das retraduções, entre 95,0\% e $100,0 \%$ de concordância nesse critério. Na outra retradução, apenas dois itens tiveram concordância inferior a esse limite: um com $85,0 \%$ e outro com $65,0 \%$ de concordância com a escala original. $\mathrm{Na}$ análise do significado geral, em ambas as retraduções, a maioria dos itens foi considerada inalterada em relação ao instrumento original. Nenhum foi avaliado como completamente alterado e apenas um item de uma das retraduções foi classificado como muito alterado (Tabela 1).

Em relação à equivalência de mensuração, tem-se que a consistência interna mensurada pelo Alpha de Cronbach para os 203 casos que fizeram parte do pré-teste evidenciou bom resultado: 0,85. Utilizando-se a amostra total de adolescentes (997), verificou-se resultado simi$\operatorname{lar}(0,80)$.

Os índices de confiabilidade intra-observador mostraram-se também significativos. $\mathrm{O}$ kappa oscilou entre discreto e moderado, com intervalo de confiança de $95 \%$, conforme mostra a Tabela 2 . O valor do coeficiente de correlação intraclasse da escala de resiliência foi de
0,746 , com intervalo de confiança entre $0,624 \%$ e $0,829 \%$.

Refletindo sobre a validade do instrumento, os três profissionais solicitados a fornecer uma opinião consensual sobre a adequação dos itens do instrumento na mensuração da resiliência confirmaram que esta tem abrangência teórico-conceitual nessa escala, dando suporte à validade de conteúdo dessa versão em português do instrumento. Mediante revisão bibliográfica e suas experiências individuais nessa área de conhecimento, consideraram os itens apropriados para medir esse conceito em adolescentes escolares.

No que se refere à validade de constructo, a escala de resiliência mostrou-se correlacionada com alguns conceitos teoricamente relevantes na literatura sobre o tema (Tabela 3). Apresentou correlação direta e significativa com autoestima, supervisão familiar, satisfação de vida e apoio social. Correlacionou-se inversamente com a escala que avalia violência psicológica.

Os resultados da análise fatorial para os 997 alunos mostraram sete fatores no estudo atual, totalizando $59,9 \%$ de percentagem acumulada da variância explicada pelos fatores na rotação ortogonal varimax e $51,1 \%$ na rotação oblíqua oblimin. Optou-se por manter no estudo fatores que explicassem a variância total em mais de $5 \%$, seguindo a mesma estratégia utilizada pelos autores da escala. Com esse critério, foram encontrados três fatores, que explicaram $32,8 \%$ da variância na rotação oblimim. A Tabela 4 mostra a carga fatorial de cada item em relação aos três fatores encontrados nesse estudo.

Esses resultados não foram similares àqueles encontrados por Wagnild \& Young ${ }^{8}$. Os dois fatores ("competência pessoal" e "aceitação de si mesmo e da vida") originalmente encontrados por esses autores ficaram distribuídos da seguinte forma no presente estudo: o primeiro fator agrupou sete itens de "competência pessoal" e sete de "aceitação de si mesmo e da vida"; no segundo fator predominaram os itens referentes a "aceitação de si mesmo e da vida", com apenas dois itens de "competência pessoal”; o terceiro fator agrupou apenas itens de “competência pessoal”.

Utilizando o método da rotação ortogonal varimax com normalização de Kaiser, o mesmo número de fatores foi encontrado. Os três fatores obtidos explicaram $39,9 \%$ da variância. A Tabela 5 mostra a carga fatorial de cada item em relação aos três fatores encontrados no presente estudo.

Comparando-se os resultados obtidos na rotação oblimim com os obtidos na varimax, verifica-se que o primeiro fator manteve-se subs- 
Equivalência semântica entre a versão original em inglês e a versão em português da escala de resiliência.

\begin{tabular}{|c|c|c|c|c|c|c|c|c|}
\hline $\begin{array}{l}\text { Formulário original } \\
\text { (inglês) }\end{array}$ & Tradução & $\begin{array}{l}\text { Versão } 1 \\
\text { Retradução }\end{array}$ & A1 & A2 & Tradução & $\begin{array}{l}\text { Versão } 2 \\
\text { Retradução }\end{array}$ & A1 & A2 \\
\hline $\begin{array}{l}\text { 1. When I make } \\
\text { plans I follow } \\
\text { through with them }\end{array}$ & $\begin{array}{l}\text { Quando eu faço } \\
\text { planos, eu prossigo } \\
\text { com eles (até o fim) } \\
\text { (eu corro atrás deles) }\end{array}$ & $\begin{array}{l}\text { When I make plans, } \\
\text { I follow then until the } \\
\text { end (I go after then) }\end{array}$ & $100 \%$ & IN & $\begin{array}{l}\text { Quando eu faço } \\
\text { planos, eu persisto } \\
\text { com eles }\end{array}$ & $\begin{array}{l}\text { When I make plans } \\
\text { I persist with them }\end{array}$ & $100 \%$ & IN \\
\hline $\begin{array}{l}\text { 2. I usually manage } \\
\text { one way or another }\end{array}$ & $\begin{array}{l}\text { Eu costumo lidar com } \\
\text { os problemas de uma } \\
\text { forma ou de outra }\end{array}$ & $\begin{array}{l}\text { I tend to deal with my } \\
\text { problems one way } \\
\text { or another }\end{array}$ & $100 \%$ & IN & $\begin{array}{l}\text { Eu normalmente lido } \\
\text { com os problemas de } \\
\text { uma forma ou de outra }\end{array}$ & $\begin{array}{l}\text { I normally deal with } \\
\text { problems in any way }\end{array}$ & $100 \%$ & IN \\
\hline $\begin{array}{l}\text { 3. I am able to } \\
\text { depend on myself } \\
\text { more than anyone } \\
\text { else }\end{array}$ & $\begin{array}{l}\text { Eu sou capaz de } \\
\text { depender de mim } \\
\text { mais do que qualquer } \\
\text { outra pessoa (contar } \\
\text { comigo) }\end{array}$ & $\begin{array}{l}\text { I am capable of } \\
\text { depending on myself } \\
\text { more than on anybody } \\
\text { else (count on me) }\end{array}$ & $100 \%$ & PA & $\begin{array}{l}\text { Eu sou capaz de } \\
\text { depender de mim } \\
\text { mesmo mais do que } \\
\text { qualquer outro }\end{array}$ & $\begin{array}{l}\text { I'm able to depend } \\
\text { on me more than } \\
\text { anyone else }\end{array}$ & $100 \%$ & PA \\
\hline $\begin{array}{l}\text { 4. Keeping interested } \\
\text { in things is important } \\
\text { to me }\end{array}$ & $\begin{array}{l}\text { Manter interesse nas } \\
\text { coisas é importante } \\
\text { para mim (pelas coisas) }\end{array}$ & $\begin{array}{l}\text { Keeping myself } \\
\text { interested on things } \\
\text { is important to me }\end{array}$ & $100 \%$ & IN & $\begin{array}{l}\text { Manter o interesse nas } \\
\text { coisas é importante } \\
\text { para mim }\end{array}$ & $\begin{array}{l}\text { Keeping the interest } \\
\text { in things is important } \\
\text { for me }\end{array}$ & $100 \%$ & IN \\
\hline $\begin{array}{l}\text { 5. I can be on my } \\
\text { own if I have to }\end{array}$ & $\begin{array}{l}\text { Eu posso estar por } \\
\text { minha conta se eu } \\
\text { precisar }\end{array}$ & $\begin{array}{l}\text { I can be on my own } \\
\text { if I have to }\end{array}$ & $100 \%$ & IN & $\begin{array}{l}\text { Eu posso ficar sozinho } \\
\text { se eu precisar }\end{array}$ & $\begin{array}{l}\text { I can be alone if } \\
\text { I need }\end{array}$ & $100 \%$ & IN \\
\hline $\begin{array}{l}\text { 6. I feel proud that I } \\
\text { have accomplished } \\
\text { things in my life }\end{array}$ & $\begin{array}{l}\text { Eu sinto orgulho de } \\
\text { ter realizado coisas } \\
\text { em minha vida }\end{array}$ & $\begin{array}{l}\text { I am proud of having } \\
\text { accomplished things } \\
\text { on my life }\end{array}$ & $100 \%$ & IN & $\begin{array}{l}\text { Eu sinto orgulho por } \\
\text { ter obtido coisas em } \\
\text { minha vida }\end{array}$ & $\begin{array}{l}\text { I feel proud of } \\
\text { obtaining things } \\
\text { in my life }\end{array}$ & $95 \%$ & PA \\
\hline $\begin{array}{l}\text { 7. I usually take } \\
\text { things in stride }\end{array}$ & $\begin{array}{l}\text { Eu costumo aceitar } \\
\text { sucessos e fracassos } \\
\text { como algo comum } \\
\text { ao longo da vida }\end{array}$ & $\begin{array}{l}\text { I tend to accept } \\
\text { success and failure } \\
\text { as something common } \\
\text { through life }\end{array}$ & $65 \%$ & MA & $\begin{array}{l}\text { Eu normalmente } \\
\text { consigo as coisas sem } \\
\text { muita preocupação }\end{array}$ & $\begin{array}{l}\text { I normally obtain } \\
\text { (get) things without } \\
\text { much worry }\end{array}$ & $100 \%$ & PA \\
\hline $\begin{array}{l}\text { 8. I am friends with } \\
\text { myself }\end{array}$ & $\begin{array}{l}\text { Eu sou amigo de } \\
\text { mim mesmo }\end{array}$ & $\begin{array}{l}\text { I am friends with } \\
\text { myself }\end{array}$ & $100 \%$ & IN & $\begin{array}{l}\text { Eu sou amigo comigo } \\
\text { mesmo }\end{array}$ & $\begin{array}{l}\text { I am friendly to } \\
\text { myself }\end{array}$ & $100 \%$ & IN \\
\hline $\begin{array}{l}\text { 9. I feel that I can } \\
\text { handle many things } \\
\text { at a time }\end{array}$ & $\begin{array}{l}\text { Eu sinto que posso } \\
\text { lidar com várias } \\
\text { coisas ao mesmo } \\
\text { tempo }\end{array}$ & $\begin{array}{l}\text { I feel like I can handle } \\
\text { lots of things at the } \\
\text { same time }\end{array}$ & $100 \%$ & IN & $\begin{array}{l}\text { Eu sinto que posso } \\
\text { lidar com várias } \\
\text { coisas ao mesmo } \\
\text { tempo }\end{array}$ & $\begin{array}{l}\text { I feel I can deal with } \\
\text { lots of things at the } \\
\text { same time }\end{array}$ & $100 \%$ & IN \\
\hline 10. I am determined & Eu sou determinado & I am determined & $100 \%$ & IN & Eu sou determinado & $\begin{array}{l}\text { I am a determined } \\
\text { person }\end{array}$ & $100 \%$ & IN \\
\hline $\begin{array}{l}\text { 11. I seldom wonder } \\
\text { what the point } \\
\text { of all is }\end{array}$ & $\begin{array}{l}\text { Eu raramente procuro } \\
\text { saber o objetivo } \\
\text { das coisas }\end{array}$ & $\begin{array}{l}\text { I rarely try to know the } \\
\text { reason for things }\end{array}$ & $85 \%$ & PA & $\begin{array}{l}\text { Eu raramente penso } \\
\text { sobre a razão das } \\
\text { coisas }\end{array}$ & $\begin{array}{l}\text { I rarely thing about } \\
\text { the reason of things }\end{array}$ & $100 \%$ & PA \\
\hline $\begin{array}{l}\text { 12. I take things one } \\
\text { day at a time }\end{array}$ & $\begin{array}{l}\text { Eu faço as coisas um } \\
\text { dia de cada vez }\end{array}$ & $\begin{array}{l}\text { I do things one day } \\
\text { at a time }\end{array}$ & $100 \%$ & IN & $\begin{array}{l}\text { Eu faço as coisas um } \\
\text { dia de cada vez }\end{array}$ & $\begin{array}{l}\text { I do things one day } \\
\text { at time }\end{array}$ & $100 \%$ & IN \\
\hline $\begin{array}{l}\text { 13. I can get through } \\
\text { difficult times because } \\
\text { I've experienced } \\
\text { difficulty before }\end{array}$ & $\begin{array}{l}\text { Eu posso passar por } \\
\text { tempos difíceis } \\
\text { porque já experimentei } \\
\text { dificuldades antes }\end{array}$ & $\begin{array}{l}\text { I can go through hard } \\
\text { times because I have } \\
\text { experienced difficulties } \\
\text { before }\end{array}$ & $100 \%$ & IN & $\begin{array}{l}\text { Eu posso superar } \\
\text { momentos difíceis, } \\
\text { porque eu passei por } \\
\text { dificuldades antes }\end{array}$ & $\begin{array}{l}\text { I am able to overcome } \\
\text { difficult moments for I } \\
\text { faced difficulties before }\end{array}$ & $100 \%$ & IN \\
\hline $\begin{array}{l}\text { 14. I have self- } \\
\text { discipline }\end{array}$ & $\begin{array}{l}\text { Eu tenho auto- } \\
\text { disciplina }\end{array}$ & I have self-discipline & $100 \%$ & IN & $\begin{array}{l}\text { Eu tenho auto- } \\
\text { disciplina }\end{array}$ & I am self-disciplined & $100 \%$ & IN \\
\hline
\end{tabular}

(continua) 
Tabela 1 (continuação)

\begin{tabular}{|c|c|c|c|c|c|c|c|c|}
\hline $\begin{array}{l}\text { Formulário original } \\
\text { (inglês) }\end{array}$ & Tradução & $\begin{array}{l}\text { Versão } 1 \\
\text { Retradução }\end{array}$ & A1 & $\mathrm{A} 2$ & Tradução & $\begin{array}{l}\text { Versão } 2 \\
\text { Retradução }\end{array}$ & A1 & $\mathrm{A} 2$ \\
\hline $\begin{array}{l}\text { 15. I keep interested } \\
\text { in things }\end{array}$ & $\begin{array}{l}\text { Eu me mantenho } \\
\text { interessado nas coisas }\end{array}$ & $\begin{array}{l}\text { I keep myself interested } \\
\text { on things }\end{array}$ & $100 \%$ & IN & $\begin{array}{l}\text { Eu mantenho o } \\
\text { interesse nas coisas }\end{array}$ & $\begin{array}{l}\text { I keep interest } \\
\text { in things }\end{array}$ & $100 \%$ & IN \\
\hline $\begin{array}{l}\text { 16. I can usually find } \\
\text { something to laugh } \\
\text { about }\end{array}$ & $\begin{array}{l}\text { Eu posso } \\
\text { freqüentemente } \\
\text { encontrar algo do } \\
\text { que rir }\end{array}$ & $\begin{array}{l}\text { I can frequently find } \\
\text { things to laugh about }\end{array}$ & $100 \%$ & IN & $\begin{array}{l}\text { Eu normalmente posso } \\
\text { achar um motivo } \\
\text { para rir }\end{array}$ & $\begin{array}{l}\text { I normally can find } \\
\text { a reason to laugh }\end{array}$ & $100 \%$ & IN \\
\hline $\begin{array}{l}\text { 17. My belief in } \\
\text { myself gets me } \\
\text { through hard times }\end{array}$ & $\begin{array}{l}\text { Minha crença em mim } \\
\text { mesmo me leva } \\
\text { a atravessar tempos } \\
\text { difíceis }\end{array}$ & $\begin{array}{l}\text { My self-confidence } \\
\text { takes me through } \\
\text { hard times }\end{array}$ & $100 \%$ & IN & $\begin{array}{l}\text { Minha crença em mim } \\
\text { mesmo me faz superar } \\
\text { momentos difíceis }\end{array}$ & $\begin{array}{l}\text { My faith in myself } \\
\text { makes me overcome } \\
\text { difficult moments }\end{array}$ & $100 \%$ & IN \\
\hline $\begin{array}{l}\text { 18. In an emergency, } \\
\text { I'm someone people } \\
\text { generally can rely on }\end{array}$ & $\begin{array}{l}\text { Em uma emergência, } \\
\text { eu sou uma pessoa } \\
\text { em quem as pessoas } \\
\text { podem contar }\end{array}$ & $\begin{array}{l}\text { In an emergency, I am } \\
\text { the person that people } \\
\text { can count on }\end{array}$ & $100 \%$ & IN & $\begin{array}{l}\text { Em uma emergência, } \\
\text { eu sou alguém com } \\
\text { quem geralmente as } \\
\text { pessoas podem contar }\end{array}$ & $\begin{array}{l}\text { In an emergency I } \\
\text { am the one with } \\
\text { whom people generally } \\
\text { can count on }\end{array}$ & $100 \%$ & IN \\
\hline $\begin{array}{l}\text { 19. I can usually look } \\
\text { at a situation in a } \\
\text { number of ways }\end{array}$ & $\begin{array}{l}\text { Eu posso geralmente } \\
\text { olhar uma situação } \\
\text { em diversas maneiras }\end{array}$ & $\begin{array}{l}\text { I can usually look at a } \\
\text { situation in different } \\
\text { ways }\end{array}$ & $100 \%$ & IN & $\begin{array}{l}\text { Eu sempre olho para } \\
\text { uma situação de } \\
\text { maneiras diferentes }\end{array}$ & $\begin{array}{l}\text { I always face a } \\
\text { situation through } \\
\text { different ways }\end{array}$ & $100 \%$ & IN \\
\hline $\begin{array}{l}\text { 20. Sometimes I } \\
\text { make myself do } \\
\text { things whether I } \\
\text { want to or not }\end{array}$ & $\begin{array}{l}\text { Às vezes eu me forço } \\
\text { a fazer coisas } \\
\text { querendo ou não }\end{array}$ & $\begin{array}{l}\text { Sometimes I force } \\
\text { myself to do things, } \\
\text { whether I like it or not }\end{array}$ & $100 \%$ & IN & $\begin{array}{l}\text { Às vezes eu me } \\
\text { obrigo a fazer coisas } \\
\text { querendo ou não }\end{array}$ & $\begin{array}{l}\text { Sometimes I force } \\
\text { myself to do things }\end{array}$ & $100 \%$ & IN \\
\hline $\begin{array}{l}\text { 21. My life has } \\
\text { meaning }\end{array}$ & $\begin{array}{l}\text { Minha vida tem } \\
\text { sentido }\end{array}$ & My life has sense & $100 \%$ & IN & $\begin{array}{l}\text { Minha vida tem } \\
\text { significado }\end{array}$ & $\begin{array}{l}\text { My life has } \\
\text { a meaning }\end{array}$ & $100 \%$ & IN \\
\hline $\begin{array}{l}\text { 22. I do not dwell on } \\
\text { things that I can't do } \\
\text { anything about }\end{array}$ & $\begin{array}{l}\text { Eu não me fixo em } \\
\text { coisas as quais eu } \\
\text { não posso fazer } \\
\text { nada sobre elas }\end{array}$ & $\begin{array}{l}\text { I don't stay on things } \\
\text { that I can't do } \\
\text { anything about }\end{array}$ & $100 \%$ & IN & $\begin{array}{l}\text { Eu não fico me } \\
\text { estendendo sobre } \\
\text { coisas que eu não } \\
\text { posso fazer nada }\end{array}$ & $\begin{array}{l}\text { I do not keeping } \\
\text { talking about things } \\
\text { I can't do anything } \\
\text { about }\end{array}$ & $95 \%$ & PA \\
\hline $\begin{array}{l}\text { 23. When I'm in a } \\
\text { difficult situation, } \\
\text { I can usually find } \\
\text { my way out of it }\end{array}$ & $\begin{array}{l}\text { Quando eu estou } \\
\text { numa situação difícil, } \\
\text { eu geralmente posso } \\
\text { achar minha maneira } \\
\text { de sair dela }\end{array}$ & $\begin{array}{l}\text { When I'm in a tough } \\
\text { situation, I can usually } \\
\text { find my way out of it }\end{array}$ & $100 \%$ & IN & $\begin{array}{l}\text { Quando eu estou } \\
\text { numa situação difícil, } \\
\text { eu normalmente } \\
\text { acho uma saída }\end{array}$ & $\begin{array}{l}\text { When I am in a } \\
\text { difficult situation } \\
\text { I usually find a } \\
\text { solution to it }\end{array}$ & $100 \%$ & IN \\
\hline $\begin{array}{l}24 . \text { I have enough } \\
\text { energy to do what } \\
\text { I have to do }\end{array}$ & $\begin{array}{l}\text { Eu tenho energia } \\
\text { suficiente para fazer } \\
\text { o que eu tenho } \\
\text { que fazer }\end{array}$ & $\begin{array}{l}\text { I have enough energy } \\
\text { to do what I have to }\end{array}$ & $100 \%$ & IN & $\begin{array}{l}\text { Eu tenho energia } \\
\text { suficiente pra fazer } \\
\text { o que preciso fazer }\end{array}$ & $\begin{array}{l}\text { I have enough } \\
\text { energy to do what } \\
\text { I need to }\end{array}$ & $100 \%$ & IN \\
\hline $\begin{array}{l}\text { 25. It's okay if there } \\
\text { are people who } \\
\text { don't like me }\end{array}$ & $\begin{array}{l}\text { Tudo bem se há } \\
\text { pessoas que não } \\
\text { gostam de mim }\end{array}$ & $\begin{array}{l}\text { It's ok if there are } \\
\text { people that don't } \\
\text { like me }\end{array}$ & $100 \%$ & IN & $\begin{array}{l}\text { Tudo bem se existem } \\
\text { pessoas que não } \\
\text { gostam de mim }\end{array}$ & $\begin{array}{l}\text { It is all right if there } \\
\text { are people who do } \\
\text { not like me }\end{array}$ & $100 \%$ & IN \\
\hline
\end{tabular}

A1: avaliação/apreciação em termos do percentual de concordância do significado referencial; A2: avaliação/apreciação do significado geral segundo categorias: IN (inalterado), PA (pouco alterado), MA (muito alterado), CA (completamente alterado). 
Confiabilidade teste-reteste da versão adaptada para o português da escala de resiliência $(n=203)$.

\begin{tabular}{|c|c|c|}
\hline Itens da escala de resiliência & $\begin{array}{l}\text { Kappa } \\
\text { ponderado } \\
\text { (linear) }\end{array}$ & $\begin{array}{l}\text { Grau de } \\
\text { concordância } \\
\text { do kappa } \\
\text { ponderado }\end{array}$ \\
\hline Quando eu faço planos, eu levo eles até o fim & 0,31 & Regular \\
\hline Eu costumo lidar com os problemas de uma forma ou de outra & 0,35 & Regular \\
\hline Eu sou capaz de depender de mim mais do que qualquer outra pessoa & 0,32 & Regular \\
\hline Manter interesse nas coisas é importante para mim & 0,16 & Discreta \\
\hline Eu posso estar por minha conta se eu precisar & 0,32 & Regular \\
\hline Eu sinto orgulho de ter realizado coisas em minha vida & 0,28 & Regular \\
\hline Eu costumo aceitar as coisas sem muita preocupação & 0,37 & Regular \\
\hline Eu sou amigo de mim mesmo & 0,42 & Moderada \\
\hline Eu sinto que posso lidar com várias coisas ao mesmo tempo & 0,30 & Regular \\
\hline Eu sou determinado & 0,53 & Moderada \\
\hline Eu raramente penso sobre o objetivo das coisas & 0,28 & Regular \\
\hline Eu faço as coisas um dia de cada vez & 0,41 & Moderada \\
\hline Eu posso enfrentar tempos difíceis porque já experimentei dificuldades antes & 0,38 & Regular \\
\hline Eu sou disciplinado & 0,34 & Regular \\
\hline Eu mantenho interesse nas coisas & 0,29 & Regular \\
\hline Eu normalmente posso achar motivo para rir & 0,32 & Regular \\
\hline Minha crença em mim mesmo me leva a atravessar tempos difíceis & 0,37 & Regular \\
\hline Em uma emergência, eu sou uma pessoa em quem as pessoas podem contar & 0,35 & Regular \\
\hline Eu posso geralmente olhar uma situação em diversas maneiras & 0,21 & Regular \\
\hline Às vezes eu me obrigo a fazer coisas querendo ou não & 0,32 & Regular \\
\hline Minha vida tem sentido & 0,50 & Moderada \\
\hline Eu não insisto em coisas as quais eu não posso fazer nada sobre elas & 0,24 & Regular \\
\hline Quando eu estou numa situação difícil, eu normalmente acho uma saída & 0,34 & Regular \\
\hline Eu tenho energia suficiente para fazer o que eu tenho que fazer & 0,41 & Moderada \\
\hline Tudo bem se há pessoas que não gostam de mim & 0,37 & Regular \\
\hline
\end{tabular}

Coeficiente de correlação de Pearson entre a escala de resiliência e constructos teoricamente relevantes.

\begin{tabular}{lc}
\hline Constructos teoricamente relevantes & Coeficiente de correlação \\
\hline Itens de supervisão familiar & $0,106^{\star}$ \\
Escala de satisfação de vida & $0,374^{\star}$ \\
Escala de auto-estima & $0,327^{\star}$ \\
Escala de violência psicológica & $-0,155^{\star}$ \\
Dimensão "apoio afetivo" da escala de apoio social & $0,204^{\star}$ \\
Dimensão "apoio emocional" da escala de apoio social & $0,233^{\star}$ \\
Dimensão "apoio de informação" da escala de apoio social & $0,231^{*}$ \\
Dimensão "interação positiva" da escala de apoio social & $0,251^{*}$ \\
\hline
\end{tabular}

${ }^{*} p<0,001(n=997)$. 
Análise fatorial da versão adaptada para o português da escala de resiliência (rotação oblíqua; $\mathrm{n}=997$ ).

\begin{tabular}{|c|c|c|c|}
\hline Item & $\begin{array}{c}\text { Fator } 1 \\
\text { carga }\end{array}$ & $\begin{array}{c}\text { Fator } 2 \\
\text { carga }\end{array}$ & $\begin{array}{c}\text { Fator } 3 \\
\text { carga }\end{array}$ \\
\hline \multicolumn{4}{|l|}{ Fator 1} \\
\hline 1. Quando eu faço planos, eu levo eles até o fim & 0,61 & $-0,01$ & $-0,32$ \\
\hline 2. Eu costumo lidar com os problemas de uma forma ou de outra & 0,36 & 0,11 & $-0,19$ \\
\hline 6. Eu sinto orgulho de ter realizado coisas em minha vida & 0,55 & $-0,18$ & $-0,26$ \\
\hline 8. Eu sou amigo de mim mesmo & 0,50 & 0,17 & $-0,21$ \\
\hline 10. Eu sou determinado & 0,61 & 0,08 & $-0,38$ \\
\hline 12. Eu faço as coisas um dia de cada vez & 0,20 & $-0,15$ & $-0,14$ \\
\hline 14. Eu sou disciplinado & 0,48 & $-0,06$ & $-0,43$ \\
\hline 16. Eu normalmente posso achar motivo para rir & 0,38 & $-0,07$ & $-0,21$ \\
\hline 18. Em uma emergência, eu sou uma pessoa em quem as pessoas podem contar & 0,58 & 0,00 & $-0,21$ \\
\hline 19. Eu posso geralmente olhar uma situação de diversas maneiras & 0,45 & 0,13 & $-0,16$ \\
\hline 21. Minha vida tem sentido & 0,69 & 0,17 & $-0,20$ \\
\hline 23. Quando eu estou numa situação difícil, eu normalmente acho uma saída & 0,66 & 0,13 & $-0,15$ \\
\hline 25. Tudo bem se há pessoas que não gostam de mim & 0,09 & 0,08 & 0,01 \\
\hline 24. Eu tenho energia suficiente para fazer o que eu tenho que fazer & 0,67 & 0,03 & $-0,27$ \\
\hline \multicolumn{4}{|l|}{ Fator 2} \\
\hline 5. Eu posso estar por minha conta se eu precisar & 0,05 & 0,68 & $-0,20$ \\
\hline 7. Eu costumo aceitar as coisas sem muita preocupação & 0,11 & 0,71 & 0,03 \\
\hline 9. Eu sinto que posso lidar com várias coisas ao mesmo tempo & 0,29 & 0,47 & $-0,26$ \\
\hline 11. Eu raramente penso sobre o objetivo das coisas & $-0,18$ & 0,22 & 0,01 \\
\hline 13. Eu posso enfrentar tempos difíceis porque já experimentei dificuldades antes & 0,10 & 0,14 & $-0,09$ \\
\hline 22. Eu não insisto em coisas as quais eu não posso fazer nada sobre elas & 0,07 & 0,11 & 0,08 \\
\hline \multicolumn{4}{|l|}{ Fator 3} \\
\hline 3. Eu sou capaz de depender de mim mais do que qualquer outra pessoa & 0,16 & 0,18 & $-0,22$ \\
\hline 4. Manter interesse nas coisas é importante para mim & 0,17 & 0,03 & $-0,83$ \\
\hline 15. Eu mantenho interesse nas coisas & 0,25 & 0,11 & $-0,84$ \\
\hline 17. Minha crença em mim mesmo me leva a atravessar tempos difíceis & 0,32 & 0,08 & $-0,33$ \\
\hline 20. Às vezes eu me obrigo a fazer coisas querendo ou não & $-0,04$ & 0,08 & $-0,23$ \\
\hline
\end{tabular}

tancialmente intacto nas duas versões (14 itens na rotação oblimim e 15 na varimax), com algumas poucas exceções (itens 11 e 25 se transferiram do fator 1 - oblimim - para o 2 - varimax; item 11 se transferiu do fator 2 - oblimim para o 1 - varimax). Já o segundo e o terceiro fator mostraram-se mais híbridos, notando-se uma tendência de transferir do fator 2 para o 3 e vice-versa, com resultados distintos.

Ao se fazer uma interpretação do agrupamento dos fatores que resultaram da rotação ortogonal varimax, abdicou-se da classificação original de competência pessoal e aceitação de si e da vida, optando-se por compreender o primeiro e principal fator, que explicou $20,6 \%$ da variância total, como itens que indicam resolução de ações e valores (que dão sentido à vida, por exemplo, amizade, realização pessoal, satisfação e significado da vida). O segundo fator, que explicou $6,7 \%$ da variância total, agrupou itens que transmitem idéia de independência e determinação. No terceiro e último fator, estão os itens indicativos de auto-confiança e capacidade de adaptação a situações, explicando 5,5 \% da variância total dos fatores.

\section{Discussão}

No que se refere à equivalência funcional da escala de resiliência, constata-se um satisfatório grau de equivalência conceitual, de itens, semântica, operacional e de mensuração entre a versão original e a brasileira, embora algumas questões mereçam ser salientadas. Apesar das dificuldades, os resultados da versão adaptada parecem 
Análise fatorial da versão adaptada para o português da escala de resiliência (rotação ortogonal; $n=997$ ).

\begin{tabular}{|c|c|c|c|}
\hline Item & $\begin{array}{c}\text { Fator } 1 \\
\text { carga }\end{array}$ & $\begin{array}{c}\text { Fator } 2 \\
\text { carga }\end{array}$ & $\begin{array}{c}\text { Fator } 3 \\
\text { carga }\end{array}$ \\
\hline \multicolumn{4}{|l|}{ Fator 1} \\
\hline 1. Quando eu faço planos, eu levo eles até o fim & 0,58 & 0,15 & 0,12 \\
\hline 2. Eu costumo lidar com os problemas de uma forma ou de outra & 0,32 & 0,04 & 0,17 \\
\hline 6. Eu sinto orgulho de ter realizado coisas em minha vida & 0,54 & 0,11 & $-0,10$ \\
\hline 7. Eu costumo aceitar as coisas sem muita preocupação & 0,08 & $-0,07$ & 0,05 \\
\hline 8. Eu sou amigo de mim mesmo & 0,48 & 0,06 & $-0,12$ \\
\hline 10. Eu sou determinado & 0,59 & 0,21 & 0,06 \\
\hline 11. Eu raramente penso sobre o objetivo das coisas & $-0,20$ & 0,04 & 0,02 \\
\hline 12. Eu faço as coisas um dia de cada vez & 0,17 & 0,09 & 0,00 \\
\hline 14. Eu sou disciplinado & 0,47 & 0,32 & 0,14 \\
\hline 16. Eu normalmente posso achar motivo para rir & 0,37 & 0,11 & 0,07 \\
\hline 18. Em uma emergência, eu sou uma pessoa em quem as pessoas podem contar & 0,57 & 0,04 & 0,25 \\
\hline 19. Eu posso geralmente olhar uma situação de diversas maneiras & 0,42 & $-0,02$ & 0,39 \\
\hline 21. Minha vida tem sentido & 0,70 & 0,05 & $-0,16$ \\
\hline 23. Quando eu estou numa situação difícil, eu normalmente acho uma saída & 0,64 & $-0,04$ & 0,26 \\
\hline 24. Eu tenho energia suficiente para fazer o que eu tenho que fazer & 0,64 & 0,07 & 0,16 \\
\hline \multicolumn{4}{|l|}{ Fator 2} \\
\hline 4. Manter interesse nas coisas é importante para mim & 0,13 & 0,82 & $-0,02$ \\
\hline 5. Eu posso estar por minha conta se eu precisar & 0,00 & 0,14 & 0,02 \\
\hline 15. Eu mantenho interesse nas coisas & 0,21 & 0,81 & 0,14 \\
\hline 25. Tudo bem se há pessoas que não gostam de mim & 0,06 & $-0,10$ & $-0,07$ \\
\hline \multicolumn{4}{|l|}{ Fator 3} \\
\hline 3. Eu sou capaz de depender de mim mais do que qualquer outra pessoa & 0,10 & 0,10 & 0,17 \\
\hline 9. Eu sinto que posso lidar com várias coisas ao mesmo tempo & 0,24 & 0,14 & 0,33 \\
\hline 13. Eu posso enfrentar tempos difíceis porque já experimentei dificuldades antes & 0,05 & $-0,02$ & 0,77 \\
\hline 17. Minha crença em mim mesmo me leva a atravessar tempos difíceis & 0,28 & 0,21 & 0,54 \\
\hline 20. Às vezes eu me obrigo a fazer coisas querendo ou não & $-0,06$ & 0,20 & 0,39 \\
\hline 22. Eu não insisto em coisas as quais eu não posso fazer nada sobre elas & 0,06 & $-0,10$ & 0,11 \\
\hline
\end{tabular}

apontar para uma boa aplicação e entendimento do instrumento na amostra investigada.

Quanto à equivalência de itens e semântica, optou-se por permanecer com todos os itens na versão em português, conservando-se os poucos que mostraram menos consistência pelas seguintes razões: comparabilidade com os estudos internacionais; manutenção de todas as áreas temáticas que constituem a resiliência; realização de um estudo exploratório que, posteriormente, poderá ser complementado com abordagens em profundidade, à medida que se tiver maior conhecimento sobre o tema.

No que tange à confiabilidade intra-observador, medida pelo kappa, constatou-se a variação entre discreta e moderada, com a maioria dos itens considerados de concordância regular de acordo com os critérios estabelecidos por Landis \& Koch 18. Esse resultado - não ideal - opõe-se à concordância das respostas globais dos alunos ao instrumento, mensurada pelo coeficiente de correlação intraclasse que indicou uma forte concordância nos dois momentos. Essa discordância pode ser influenciada pela forma como as opções de respostas da escala são colocadas (existem três níveis de concordância, três de discordância e um nível situado entre eles, indicando não concordar com a afirmativa nem discordar dela). Essa distribuição facilita pequenas mudanças de opinião em dois momentos distintos, não implicando respostas totalmente opostas como sugerido através da boa concordância entre as respostas do indivíduo à escala como um todo.

Os escores de alpha de Cronbach encontrados na versão brasileira se assemelham ao rela- 
tado por Wagnild \& Young $8(0,91)$ e Aroian et al. $5(0,87)$, indicando boa consistência interna do instrumento.

As correlações entre escores da escala de resiliência e constructos, teoricamente ligados ao comportamento resiliente, foram na direção esperada e apresentaram-se estatisticamente significantes $(\mathrm{p}<0,01)$. A maior correlação foi com a escala de satisfação de vida de Diener et al. 20, na qual o sujeito julga o quanto está satisfeito com a sua vida, independentemente daquilo que vivencia. Outros autores têm demonstrado a relação entre a escala de resiliência com atributos emocionais. Aroian et al. 5 constataram que elevada resiliência se correlaciona com baixa somatização e depressão, bem como com maior saúde física, determinação, sensação de bem-estar e habilidade de lidar com problemas $(\mathrm{p}<0,001)$.

Um ponto delicado se refere ao resultado das análises fatoriais. Não foram encontrados apenas os dois fatores observados na escala original. Ainda, os três fatores obtidos no presente estudo não distinguiram nitidamente competência pessoal e satisfação de si e da vida, tomando-se a decisão de distingui-los segundo categorias teóricas que constituem a resiliência: capacidade de resolver situações, valores, independência, determinação, autoconfiança e adaptação. Algumas hipóteses podem ser atribuídas a essa diferença.

Primeiramente, deve-se ressaltar que a escala de resiliência foi originalmente construída com uma amostra de mulheres e somente aplicada em população adulta. Ainda não há como aferir se a resiliência se manifesta da mesma forma em diferentes faixas etárias. Isto é, não há, até o momento, como identificar se para adolescentes há distinção tão significativa entre "competência pessoal" e "valorização de si e da vida”. Por outro lado, vários dos conceitos definidos no presente estudo estão teoricamente relacionados entre si, razão pela qual a formação dos fatores mostra algumas ambigüidades. Tomando-se como exemplo o fator de resolução de ações, tem-se que os itens 1 e 10 podem também ser identificados como determinação e os itens 7 e 11 podem ser compreendidos como adaptação a problemas.

Outra possibilidade é que os dados psicométricos existentes internacionalmente, testados em populações adultas de culturas muito distintas 5,8 , tenham se organizado na análise fatorial de forma diferente por problemas de entendimento do conceito, ainda não totalmente cristalizado e compreendido. Nesse caso, a escala tal qual se apresenta não seria realmente indicada em culturas diversas, merecendo adaptações mais drásticas, além de exaustivos estudos comparativos.

Apresenta-se a favor de diferentes abordagens conceituais entre culturas o fato de que Aroian et al. 5 sofreram problema similar ao adaptarem a mesma escala para uma amostra de 450 imigrantes russos adultos. Tomaram rumo diferente, optando por manter os dois fatores originalmente propostos, com apenas 12 itens da escala. Interessante ressaltar que os pesquisadores russos encontraram pouca equivalência em relação aos mesmos itens observados na adaptação brasileira. Argumentaram que tais itens não teriam significação cultural para os imigrantes estudados.

O conceito de resiliência vem sendo construído apenas nos dias atuais. Esse campo da ciência foi recentemente inaugurado e, embora muitos autores tenham se dedicado ao estudo dos fatores que levam o sujeito a lidar positivamente com dificuldades $1,25,26$, existe um caminho a ser percorrido até que se chegue a hipóteses mais concretas. Contudo, mesmo em face da incompletude, a resiliência se instaurou no campo da promoção da saúde, discutindo-se hoje a ampliação do conceito para além dos indivíduos, por intermédio de expressões como escolas resilientes ou comunidades resilientes 27 .

O caráter inédito deste trabalho decorre do fato de que há poucas investigações priorizando métodos de adaptação transcultural de escalas e índices psicométricos, especialmente em escalas com temática similar à proposta no presente artigo. Mesmo em língua estrangeira, ainda são poucos os estudos desenvolvidos sobre a questão. Cumpre ainda ressaltar a inexistência de estudos epidemiológicos com escalas de resiliência envolvendo adolescentes (apenas um texto foi localizado, em japonês 7).

Em benefício da utilização da versão brasileira da escala de resiliência, destacam-se os bons resultados da validade de constructo, indicando capacidade de discriminar vários atributos relacionados à resiliência. Acredita-se que novos estudos possam contribuir para se compreender melhor a organização interna da escala em fatores, possibilitando, também, o refinamento do instrumento e, possivelmente, sua redução. Além das várias limitações observadas nas versões da escala de resiliência, o próprio autor do instrumento revela o fato de que este não possui questões para abordar teoricamente baixa resiliência. Apenas os indivíduos resilientes seriam aferidos pelo instrumento.

$\mathrm{Na}$ realidade, as limitações e incertezas da escala de resiliência refletem as instabilidades 
no conhecimento sobre o tema. A ausência de outros instrumentos de aferição dificulta qualquer análise mais aprofundada. Apenas com a disseminação do conhecimento sobre o tema e com a adesão de novos pesquisadores se poderá chegar a uma escala de resiliência mais estável e replicável, contribuindo não apenas para o desenvolvimento metodológico, como também, principalmente, para o conhecimento, divulgação e promoção dos recursos necessários para que as pessoas superem as dificuldades vivenciadas ao longo da vida.

\section{Resumo}

Busca-se apresentar os resultados da adaptação transcultural para o português da escala de resilência de Wagnild \& Young e da avaliação psicométrica desta. A escala foi adaptada para uma amostra de escolares dos ensinos fundamental e médio da rede pública de ensino do Município de São Gonçalo, Rio de Janeiro, Brasil. São apresentados dados do estudo-piloto, com 203 alunos entrevistados em dois momentos consecutivos (teste-reteste), bem como do total de alunos investigados na pesquisa (977). Quanto à adaptação transcultural, foram encontrados bons resultados na equivalência semântica dos itens, tanto para o significado geral,quanto para o referencial. O alfa de Chronbach encontrado foi de 0,85 no pré-teste e 0,80 na amostra total. Na confiabilidade intra-observador, verificou-se que o kappa situou-se entre regular e moderado e o coeficiente de correlação intraclasse foi de $0,746(p=0,000)$. Análise fatorial indicou três fatores não totalmente homogêneos e diferenciados dos achados pelo autor da escala. Quanto à validade de constructo, nota-se correlação direta e significativa com auto-estima, supervisão familiar, satisfação de vida e apoio social. Verificou-se correlação inversa com a escala que avalia violência psicológica.

Adaptação; Reprodutibilidade de Resultados; Questionários

\section{Colaboradores}

R. P. Pesce, S. G. Assis e J. Q. Avanci foram responsáveis pelo processo de coleta e análise dos dados da pesquisa, pelo acompanhamento da etapa de adaptação transcultural da escala de resiliência e pela discussão teórica sobre o tema. N. C. Santos, J. V. Malaquias e R. Carvalhaes participaram do armazenamento dos dados quantitativos e da realização das análises estatísticas inseridas no artigo. R. P. Pesce elaborou o texto do artigo e S. G. Assis contribuiu com a constante supervisão da elaboração do texto. 


\section{Referências}

1. Rutter M. Psychosocial resilience and protective mechanisms. Am J Orthopsychiatry 1987; 57:31631.

2. Luthar SS, Zigler E. Vulnerability and competence: a review of research on resilience in childhood. Am J Orthopsychiatry 1991; 61:6-22.

3. Bloom M. Primary prevention and resilience: changing paradigms and changing lives. In: Hampton RL, Jenkins P, Gulotta TP, editors. Preventing violence in America. California: Sage; 1996. p. 87114.

4. Al-Naser F, Sandman M. Evaluation resilience factors in the face of traumatic events in Kuwait. Medical Sciences 2000; 2:111-6.

5. Aroian KJ, Schaooker-Morris N, Neary S, Spitzer A, Tran TV. Psychometric evaluation of the Russian language version of the resilience scale. J Nurs Meas 1997; 5:151-64.

6. Hunter AJ, Chandler GE. Adolescent resilience. J Nurs Scholarsh 1999; 31:243-7.

7. Oshio A, Nakaya M, Kaneco H, Nagamine S. Development and validation of an adolescent resilience scale. Japanese Journal of Counseling Science 2002; 35:57-65.

8. Wagnild GM, Young HM. Development and psychometric evaluation of resilience scale. J Nurs Meas 1993; 1:165-78.

9. Patrick DL, Sittampalam Y, Somerville SM, Carter WB, Bergner M. A cross-cultural comparison of health status values. Am J Public Health 1985; 75:1402-7.

10. Bravo M, Canino GJ, Rubio-Stipec M, WoodburyFarina M. A cross-cultural adaptation of a psychiatrc epidemiologic instrument: The diagnostic interview schedule's adaptation in Puerto Rico. Cult Med Psychiatry 1991; 15:1-18.

11. Badia X, Alonso J. Re-scaling the spanish version of the sickness impact profile: an opportunity for the assessment of cross-cultural equivalence. J Clin Epidemiol 1995; 48:949-57.

12. Herdman M, Fox-Rushby J, Badia X. A model of equivalence in the cultural adaptation of HRQol instruments: the universalist approach. Qual Life Res 1998; 7:323-35.

13. Hasselmann MH, Reichenheim ME. Adaptação transcultural da versão em português da Conflict Tactics Scales Form R (CTS-1), usada para aferir violência no casal: equivalência semântica e de mensuração. Cad Saúde Pública 2003; 19:1083-93.
14. Moraes CL. Aspectos metodológicos relacionados a um estudo sobre a violência familiar durante a gestação como fator de propensão da prematuridade do recém-nascido [Tese de Doutorado]. Rio de Janeiro: Escola Nacional de Saúde Pública, Fundação Oswaldo Cruz; 2001.

15. Moraes CL, Hasselmann MH, Reichenheim ME. Adaptação transcultural para o português do instrumento Revised Conflict Tactics Scales (CTS2), utilizado para identificar violência entre casais. Cad Saúde Pública 2002; 18:163-76.

16. Ohlsson E. Sequencial poisson sapling. J Off Stat 1998; 14:149-62.

17. Streiner DL, Norman GR. Health measurement scales: a practical guide to their development and use. Oxford: Oxford University Press; 1995.

18. Landis JR, Koch GG. The measurement of observer agreement for categorical data. Biometrics 1977; 33:159-74

19. Rosenberg M. Society and the adolescent self-image. Princeton: Princeton University Press; 1989.

20. Diener E, Emmons RA, Larsen RJ, Griffin S. The satisfaction with life scale. J Pers Assess 1985; 49:71-5.

21. Sherbourne CD, Stewart AL. The MOS social support survey. Soc Sci Med 1991; 32:705-14.

22. Pitzner JK, Drummond PD. The reliability and validity of empirically scaled measures of psychological/verbal control and physical/sexual abuse: relationship between current negative mood and a history of abuse independent of other negative life events. J Psychosom Res 1997; 2:125-42.

23. Assis SG, Avanci JQ. Labirinto de espelhos. A formação da auto-estima na infância e adolescência. Rio de Janeiro: Editora Fiocruz; 2003.

24. Rennie K. Exploratory and confirmatory rotation strategies in exploratory factor analysis. http// www.crical.net/ft/tamu/rota.htm (acessado em 16/Out/2003).

25. Garmezy N, Rutter M. Stress, coping and development in children. New York: Johns Hopkins University Press; 1988.

26. Haggerty RJ, Sherrod LR, Garmezy N, Rutter M. Stress, risk and resilience in children and adolecents: processes, mechanisms and interventions. Cambridge: Cambridge University Press; 1996.

27. Travis J, Wail M. Reflections on the crime decline: lesson for the future? Proceedings from the Urban Institute Crime Decline Forum. Washington DC: Urban Institute Justice Policy Center; 2002.

Recebido em 03/Nov/2003

Versão final reapresentada em 19/Mar/2004

Aprovado em 03/Set/2004 